\title{
Investigación del estado de flow en educación física para primaria
}

\author{
Alberto Blázquez Manzano. Universidad Camilo José Cela \\ Cristina Espigares Balboa. Universidad Camilo José Cela
}

Recepción: 17.07.2018 | Aceptado: 5.12.2018

Correspondencia a través de ORCID: Cristina Espigares

(iD 0000-0002-8586-9391

Citar: Bláquez Manzano, A. y Espigares Balboa, C. (2018). Investigación del estado de flow en educación física para Educación Primaria. ReiDoCrea, 7, 388-394.

\begin{abstract}
Resumen: Nuestro trabajo se apoya en el estudio realizado por Castaño Pérez, Navarro Patón y Basanta Camiño (2015) "sobre el tipo de motivación que predomina en los escolares de Educación Primaria hacia la Educación Física”, en la tesis doctoral de Carrasco Beltrán, "las investigaciones sobre motivación autodeterminada y estado de flow en actividades físico deportivas" y con la tesis de Jiménez Torres, "experiencias del flow en niños y jóvenes: Influencias en distintos indicadores de rendimiento y bienestar". Tenemos por objetivo analizar el estado del flujo durante actividades físicas y deportivas, en estudiantes de $6^{\circ}$ de primaria. Método: realizamos un estudio cuantitativo, a través de un cuestionario para evaluar el estado de flow de los estudiantes, dividido en 38 ítems. Los resultados se obtienen a través del programa SPSS. Tras analizarlos y seleccionar los indicadores, mostraron que existe una correlación entre flujo y aprendizaje. Resultados: la comparación de las medias de las variables que correlacionan en el modelo muestra un resultado significativo $\left(F_{2,47}=44.735 ; p=0.0\right)$ y una posibilidad de réplica de un 39\% (Beta). Las conclusiones: obtenidas se pueden sintetizar en que hay 2 variables predictoras de la auto-percepción: Sentimiento de Control y Automatización.
\end{abstract}

Palabras clave: Educación Primaria | Educación Física

\section{Research of the state of flow in Physical Education for Primary}

\begin{abstract}
Our work is based on the study carried out by Castaño Pérez, Navarro Patón and Basanta Camiño (2015) "on the type of motivation that predominates in primary school students towards physical education", in the doctoral thesis of Carrasco Beltrán, "the research on self-determined motivation and state of flow in sports physical activities "and with the thesis of Jiménez Torres," experiences of flow in children and young people: Influences in different indicators of performance and well-being ".We aim to analyze the state of the flow during physical and sports activities, in 6th grade students. Method: we conducted a quantitative study, through a questionnaire to assess the flow state of the students, divided into 38 items. The results are obtained through the SPSS program. After analyzing them and selecting the indicators, they showed that there is a correlation between flow and learning. Results: the comparison of the means of the variables that correlate in the model shows a significant result $\left(F_{2,47}=44.735 ; p=0.0\right)$ and a replication possibility of $39 \%$ (Beta). The conclusions: obtained can be summarized in that there are 2 predictors of self-perception: Control Sentiment and Automation.
\end{abstract}

Keywords: Primary Education | Physical Education

\section{Introducción}

En educación, se fomenta el concepto de sobre-aprendizaje que es un factor importante en esta técnica, explicado en el libro Experiencia Óptima. El sobreaprendizaje fomenta una concentración mental, visualizando el rendimiento deseado como algo único, una acción integrada en vez de un conjunto de acciones (Csikszentmihalyi, 2000).

El autor anteriormente mencionado meditó los principios y prácticas del llamado Método Montessori de educación, que consiste en proveer un ambiente particular, siendo este un lugar con un orden atractivo, simple, espacial con el fin de que cada elemento que lo confecciona tenga su razón de ser en el desarrollo de los alumnos. El ambiente preparado que brinda al alumnado oportunidades para comprometerse en un trabajo interesante, 
libremente elegido, que propicia prolongados períodos de concentración que no deben interrumpirse. La libertad se desarrolla dentro de límites claros que permite a los niños convivir en la micro sociedad de la clase (Csíkszentmihályi, 2000).

Los alumnos aprenden con materiales específicos, especialmente creados, que brindan las claves para descubrimiento del mundo que nos rodea, y para impulsar capacidades cognitivas esenciales. Los instrumentos utilizados han sido elaborados con el fin de que el alumno sea responsable y autónomo de su propio aprendizaje. Esta forma de enseñanza-aprendizaje tiene por objetivo que los alumnos obtengan de forma intencionada experiencias y oportunidades de flow. Csíkszentmihályi y el psicólogo Kevin Rathunde emprendieron un estudio de estudiantes de varias edades en el que se comparó los colegios Montessori con las escuelas tradicionales.

Una de las diferencias a enfatizar que encontró es, que en los colegios tradicionales, el alumnado dedica la mayor parte del tiempo a escuchar a los maestros y a tomar apuntes, una actividad altamente estructurada. Por el contrario, en los colegios Montessori, el alumnado está más horas involucrado en proyectos independientes, ya sean grupales o individuales. Este tipo de actividad dota una combinación de libertad y estructura. No es casualidad que el alumnado de los colegios Montessori valoraran a sus compañeros de forma más favorable, sus maestros y su colegio. Estaban más involucrados en sus tareas escolares, con más energía y mostraban niveles más altos de motivación intrínseca.

El presente trabajo se ha basado en una investigación sobre el tema: "investigación sobre el estado de flow en la educación física para $6^{\circ}$ curso de educación primaria". Se han utilizado artículos y referencias web. Una de las limitaciones encontradas a lo largo de la búsqueda bibliográfica es la antigüedad de parte de las publicaciones sobre el tema del flow, aún así, el sustento teórico que aportado por los libros y los artículos científicos son de vital importancia para examinar la dualidad flow destructivo - bienestar.

Por ello, estos datos irían en línea con los encontrados en revista científica Dialnet, el estudio realizado por Castaño Pérez, Navarro Patón y Basanta Camiño (2015) sobre el tipo de motivación que predomina en los escolares de Educación Primaria hacia la Educación Física". Por otro lado, en la línea de la tesis doctoral realizada por Carrasco Beltrán donde analiza en uno de los puntos de dicha tesis "las investigaciones sobre motivación auto-determinada y estado de flow en actividades físico deportivas". Otra Tesis que está ampliamente relacionada con los resultados obtenidos en mi estudio es la realizada por Jiménez Torres, sobre "experiencias del flow en niños y jóvenes: Influencias en distintos indicadores de rendimiento y bienestar".

Este trabajo se divide en diferentes partes. En primer lugar, se explicará la teoría del flow tras consultar varios artículos y páginas web; después, se examinarán los modelos, las teorías y variables que influyen en el estado del flow. A continuación, observaremos cómo se ha evaluado este estudio, a través de un cuestionario y su análisis por medio del programa SPSS. Por otro lado, el curriculum y su relación con el flow y por último, aplicaciones prácticas en Educación para la gestión del flow en las clases. 


\section{Objetivos o hipótesis}

Analizar la auto-percepción de los alumnos de Educación Primaria (en concreto $6^{\circ}$ curso del colegio Lux Mundi) durante actividades deportivas.

\section{Participantes}

En este estudio han participado 50 estudiantes de $6^{\circ}$ curso de Educación Primaria, de los cuales 18 son alumnos (36\% del total) y 32 alumnas (64\% del total), con edades comprendidas entre los 11 y los 12 años y que participaron de forma voluntaria. El estudio se ha realizado durante las dos primeras semanas del mes de Febrero de 2018 durante el horario escolar.

\section{Instrumento}

El instrumento que se ha usado para esta investigación ha sido un cuestionario de elaboración propia basado en la Flow Scale State, para evaluar el estado de Flow de los estudiantes. Este cuestionario se divide en 38 ítems, de los cuales podemos extraer 10 variables o factores, a los que se le ha añadido también la Variable $10(\mathrm{~V}-10)$ que es de auto-percepción. Dicho cuestionario podemos observarlo en los anexos página 35 , y posteriormente se puede observar la tabla de recogida de datos en el anexo 2 páginas 36 y 37 .

En cuanto al formato de respuesta del cuestionario es de una escala tipo Likert de 6 anclajes que van desde "nunca" (1) hasta "siempre" (6). También recoge datos generales, respecto a género y edad.

Los diferentes cuestionarios se han pasado de forma individual y anónima, en clases distintas de la Educación Primaria. Se les ha dejado todo el tiempo que precisasen para realizarlo y se les ha explicado que no hay preguntas correctas o incorrectas, sólo debían contestar con la frecuencia con la que han experimentado cada una de las frases que aparecen en el cuestionario, siendo 1 "nunca" y 6 "siempre".

Tras comprobar que los 50 cuestionarios estaban cumplimentados correctamente, se ha procedido a analizar los datos obtenidos y seleccionar indicadores de Flow de las personas participantes, a través de un primer análisis de correlación de Pearson y un segundo de regresión jerárquica por pasos, lo que ha permitido sacar unas conclusiones valiosas del material recogido.

Para valorar el flow en estudiantes de primaria hemos utilizado como instrumento, el cuestionario que se refleja posteriormente. $Y$ con referencia al punto mencionado ( 7.4 Variables que influyen en el estado de "flow") podemos ver las variables o factores en los que hemos dividido el cuestionario:

- V-1: Equilibrio reto-habilidad, (ítems: 1, 10, 19, 28)

- V-2: Automatismo (ítems: 2, 11, 20, 29)

- V-3: Claridad de Objetivos (ítems: $3,12,21,30$ )

- V-4: Claridad en el feedback (ítems: $4,13,22,31$ )

- V-5: Concentración (ítems: 5, 14, 23, 32)

- V-6: Sentimiento de Control (ítems: 6, 15, 24, 33)

- V-7: Pérdida de la auto-conciencia (ítems: $7,16,25,34$ ) 
- V-8: Distorsión del tiempo (ítems: 8, 17, 26, 35)

- V-9: Experiencia autotélica. (ítems: 9, 18, 27, 36)

- V-10: Auto-percepción de flow. (ítems: 37, 38)

El análisis de fiabilidad es de 0.951 siendo el máximo 1.

\section{Resultados}

El análisis de correlación de Pearson muestra que todas las dimensiones correlacionan entre ellas y con la auto-percepción de Flow que tienen los participantes (Tabla 1).

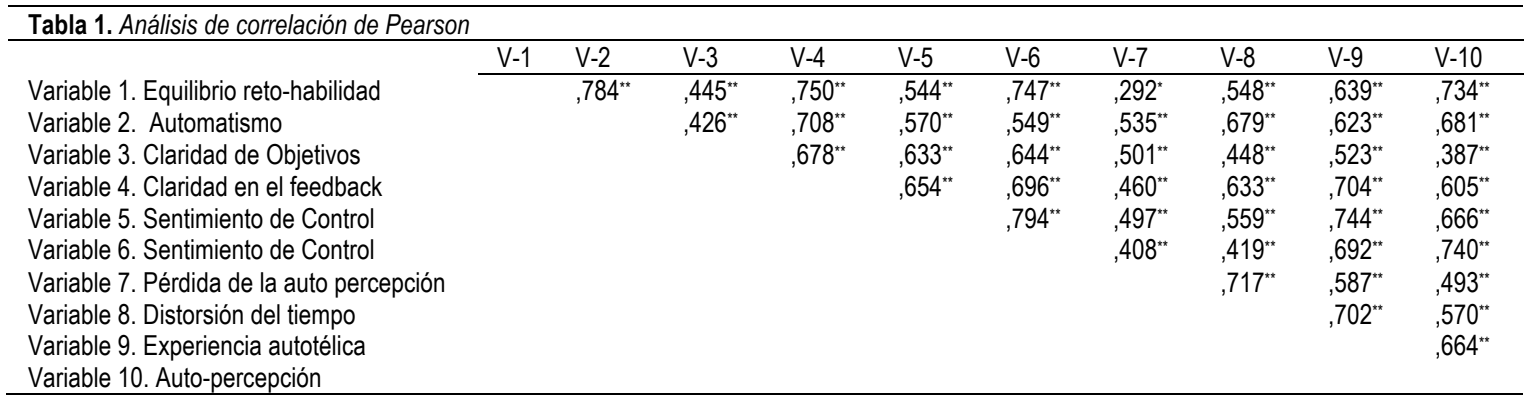

En cuanto a la regresión lineal revela que existen 2 variables -Variable de Sentimiento de Control y Variable de Automatismo- que son predictoras de la variable dependiente -Variable de auto-percepción- de Flow.

\begin{tabular}{lcccll}
\hline \multicolumn{6}{c}{ Tabla 2. Regresión lineal ANOVA } \\
\hline Modelo & Suma de cuadrados & gl & Media cuadrática & F & Sig. \\
\hline Regresión & 79,053 & 2 & 39,526 & 44,735 & $\mathbf{0 , 0 0 0}$ \\
Residual & 41,527 & 47 & 0,884 & & \\
Total & 120,580 & 49 & & & \\
\hline a. Variable dependiente: Variable10 & & & & \\
c. Variables predictoras: (Constante), Variable 6, Variable 2 & & \\
\hline
\end{tabular}

La comparación de las medias de las variables que correlacionan en el modelo muestra un resultado significativo $\left(F_{2,47}=44.735 ; p=0.0\right)$ y una posibilidad de réplica de un $39 \%$ (Beta). Es decir, se puede observar que si volviésemos a repetir el estudio con una muestra similar, la probabilidad de la réplica de los datos sería de un $39 \%$.

\begin{tabular}{|c|c|c|c|c|c|}
\hline \multicolumn{6}{|c|}{ Coeficientes $^{a}$} \\
\hline Modelo & Coeficientes & no estandarizados & Coeficientes tipificados & $T$ & Sig. \\
\hline V-6;V-2 & $\begin{array}{c}\mathrm{B} \\
0,171\end{array}$ & $\begin{array}{c}\text { Error típ. } \\
\mathbf{0 , 0 4 4}\end{array}$ & $\begin{array}{c}\text { Beta } \\
\mathbf{0 , 3 9 4}\end{array}$ & 3,851 &, 000 \\
\hline
\end{tabular}

Utilizamos las variables predictoras $\mathrm{V}-6$ y $\mathrm{V}-2$, para realizar el estudio de la variable que nos interesa en nuestra investigación -V-10, la auto-percepción-. Entendemos con estas tablas que la variable 10 es la dependiente a las dos nombradas anteriormente.

\begin{tabular}{|c|c|c|c|c|}
\hline \multicolumn{5}{|c|}{ Resumen del modeloc } \\
\hline Modelo & $\mathrm{R}$ & R cuadrado & R cuadrado corregida & Error típ. de la estimación \\
\hline & $0,810^{b}$ & 0,656 & 0,641 & 0,93998 \\
\hline
\end{tabular}


Por último, se expone el gráfico de la prueba de normalidad en el cual se puede comprobar cómo los datos cumplen con la probabilidad de acumulación esperada y observada linealmente.

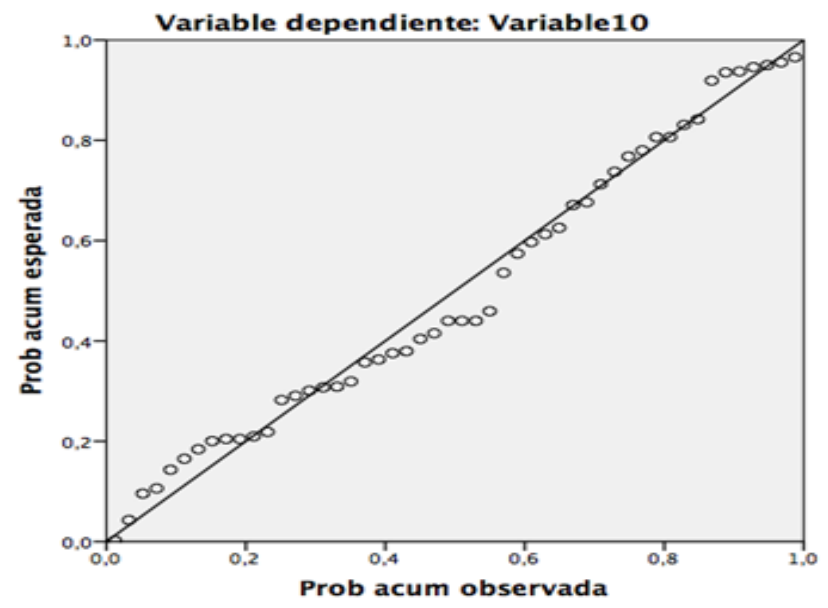

Figura 5: Prueba de normalidad, para la comprobar datos.

Este gráfico representa cada uno de los resultados obtenidos sobre la variable 10 de auto-percepción, donde se muestra que los datos obtenidos no están dispersos y mantienen una figura cerca de la línea de la representación normal de éstos. Con lo cual, nos lleva a predecir que el estudio es fiable y hemos obtenido los resultado positivos, ya que sí los diferentes puntos estuviesen muy dispersos el estudio realizado no sería óptimo y no cumpliríamos el objetivo marcado.

\section{Discusión}

Los resultados obtenidos en nuestro estudio muestran que existe correlación entre todas las dimensiones con la que nos interesa que es la del flow en los estudiantes de $6^{\circ}$ curso de primaria. De esta manera, comprobamos que es importante tener en cuenta todas las dimensiones a la hora de mejorar cualquier programa de Educación Física.

Después de realizar la regresión lineal para comprobar que modelo es el predictor de la décima variable -auto-percepción-, comprobamos que el es más significativo en este estudio nos indica que debemos de seguir las dos variable 2 y 6 , por las cuales estas dos dimensiones son predictoras. Por lo tanto, si se mejoran estas dos dimensiones en el alumnado, su desarrollo del flow va a mejorar.

A continuación, en los análisis realizados, la réplica es de un $39 \%$, dato interesante porque en ciencias sociales los datos obtenidos de las réplicas en los estudios suelen ser menores. En este caso es interesante porque después de hacerse el estudio se ha mostrado que ese modelo se puede replicar con bastante éxito. Por ello, seguiríamos las mismas actuaciones que hemos llevado a cabo saliendo del marco determinado de las teorías de flow y sobre todo aplicado a los que es la educación física.

Finalmente para concluir la aplicación de cualquier programa de flow en educación física va a beneficiar tanto al alumnado como al profesorado y además a las personas externas al centro educativo como pueden ser las familias. 
En este estudio hemos de resaltar que existen limitaciones porque es una población pequeña y por la falta de tiempo.

Hemos de valorar de manera objetiva el estudio, por tanto, hay que destacar como límite la muestra, ya que tan solo plasma a los estudiantes de $6^{\circ}$ de primaria del Colegio Lux Mundi de Cájar (Granada). El total de la muestra es de 50 estudiantes encuestados, aunque solo se puede interpretar desde el punto de vista del alumnado participante de $6^{\circ}$ de Primaria.

La prospectiva que podríamos emplear es este estudio a toda la etapa educativa es aumentar la muestra y la fiabilidad del estudio.

Por otro lado, podríamos hacer el estudio en otros centros educativos, puesto que podríamos comparar: niveles socioeconómicos, características de colegios públicos... para no sesgar la muestra en un perfil específico.

También como prospectiva, se podría adaptar el cuestionario, eliminando aquellos ítems que en este primer estudio no han obtenido resultados significativos o concluyentes, en la relación a las variables. Para enriquecer así, en un futuro los resultados.

Ha sido necesario el enfoque cualitativo por la recopilación y el trabajo con los datos. Y por supuesto, en último lugar, para poder exponer gráficamente los resultados obtenidos. Además, es una buena forma la de utilizar el instrumento para la recopilación rápida y sesgada de los datos.

\section{Referencias}

Aznar, I., Cáceres, M., e Hinojo, F. (2007). Estudio de la Violencia y Conflictividad Escolar en las Aulas de Educación Primaria a través de un Cuestionario de Clima de Clase: El Caso de las Provincias de Córdoba y Granada (España). REICE. Revista Iberoamericana sobre Calidad, Eficacia y Cambio en Educación, 5(1),164-177.

Bisquerra, R. y Pérez, N. (2007). Las competencias emocionales. Educación XXI, 10, 61-82.

Carrasco,H. (2015).Efectos de los Juegos Reducidos Basados en el Futbol sobre Indicadores de Saluden Adolescentes Chilenos. (tesis doctoral). Universidad de Granada, España

Castaño, M. Navarro, R. y Basanta, S. (2015). “¿Qué tipo de motivación predomina en los escolares de educación primaria en los escolares hacia la educación física". ISSN.989-8304.

Csikszentmihalyi, M. (1975). Beyond borendom and anxiety. San Fancisco: Jossey-Bass.

Csikszentmihalyi, M. (1990). Fluir: una psicología de la felicidad. Barcelona: Kairós.

Csikzentmihalyi, M. (1990). Flow: The psychology of optimal experience. New York: Harper \& Rowe.
Csikszentmihalyi, M. y Csikszentmihalyi, IS. (1998). Experiencia óptima. Estudios psicológicos del flujo en la conciencia. Bilbao: Desclée De Brouwer.

Csikszentmihalyi, M., \& Larson, R. (1984). Being Adolescent: Conflict and Growth in the Teenage Years. New York: Basic Books.

Cuadra, H. y Florenzano, R. (2003). El Bienestar Subjetivo: Hacia una Psicología Positiva. Revista de Psicología Universidad de Chile, 12, 83-96.

García Calvo, T., Jiménez, R., Santos-Rosa, FJ., Reina, R. y Cervelló, E. (2008) Psychometric properties of the spanish version of the Flow State Scale. The Spanish Journal of Psychology, 11, 660-669.

Goyanes, JM. (2008). El método Montessori. Recuperado de http://www.fundacionmontessori.org/metodomontessori.htm

Jackson, Thomas, Marsh \& Smethurst (2001). Relationships between Flow, Self-Concept, Psychological Skills, and Performance. Journal of Applied Sport Psychology, 13(2), 129-153.

Jackson, SA., \& Eklund, RC. (2002). Assessing flow in physical activity: the flow state scale-2 and dispositional flow scale-2. Journal of Sport and Exercise Psychology, 24, 133-150. 
Jiménez,M. (2015).Experiencias de flow en niños y jóvenes: Influencias en distintos indicadores de rendimiento y bienestar. (Tesis doctoral). Universidad de Granada. Facultad de Psicología, España.

Kimiecik, JC., \& Stein, GL. (1992). Examinig flow experiences in sport contexts: Conceptual issues and methodological concerns. Journal of Applied Sport Psychology, 144-160.

Moreno, JA., Alonso, N., Martínez Galindo, C. y Cervelló, E. (2005). Motivación, disciplina, coeducación y estado de flow en educación física: Diferencias según la satisfacción, la práctica deportiva y la frecuencia de práctica. Cuadernos de Psicología del Deporte, 5, 1-2, 225-243.
Papaioannou, A. (1994). Development of a questionnaire to measure achievement orientations in physical education. Research Quarterly for Exercise and Sport, 65, 1-20.

Sheldon, KM., \& King, L. (2001). Why positive psychology is necessary. American Psychologist, 56, 2C.M.

Tardy, C., \& Snyder, B. (2004). That's why I do it: flow and EFL teachers practices. ELT Journal, 58, 118128.

Young, JA. (1999). Professional tennis players in flow: Flow theory and reversal theory perspectives. Unpublished doctoral thesis. Monash University, Melbourne. 